\title{
A New Extended Uniform Distribution
}

\author{
K. K. Sankaran ${ }^{1}$, K. Jayakumar ${ }^{2}$ \\ ${ }^{1}$ Department of Statistics, Sree Narayana College, Kerala, India \\ ${ }^{2}$ Department of Statistics, University of Calicut, Kerala, India
}

Email address:

snsankaran08@gmail.com (K. K. Sankaran),jkumar19@rediffmail.com (K. Jayakumar)

\section{To cite this article:}

K. K. Sankaran, K. Jayakumar. A New Extended Uniform Distribution. International Journal of Statistical Distributions and Applications. Vol. 2, No. 3, 2016, pp. 35-41.doi: 10.11648/j.ijsd.20160203.12

Received: October 14, 2016; Accepted: November 7, 2016; Published: December 5, 2016

\begin{abstract}
We introduce a new family of distributions using truncated the Discrete Mittag- Leffler distribution. It can be considered as a generalization of the Marshall-Olkin family of distributions. Some properties of this new family are derived. As a particular case, a three parameter generalization of Uniform distribution is given special attention. The shape properties, moments, distributions of the order statistics, entropies are derived and estimation of the unknown parameters is discussed. An application in autoregressive time series modeling is also included.
\end{abstract}

Keywords: Discrete Mittag-Leffler Distribution, Entropy, Marshall-Olkin Family of Distributions, Maximum Likelihood, Random Variate Generation, Truncated Negative Binomial Distribution, Uniform Distribution

\section{Introduction}

Many researchers are interested in search that introduces new families of distributions or generalization of distributions which can be used to describe the lifetimes of some devices or to describe sets of real data. Exponential, Rayleigh, Weibull and linear failure rate are some of the important distributions widely used in reliability theory and survival analysis. However, these distributions have a limited range of applicability and cannot represent all situations found in application. For example, although the exponential distribution is often described as flexible, its hazard function is constant. The limitations of standard distributions often arouse the interest of researchers in finding new distributions by extending ones. The procedure of expanding a family of distributions for added flexibility or constructing covariates models is a well known technique in the literature.

Uniform distribution is regarded as the simplest probability model and is related to all distributions by the fact that the cumulative distribution function, taken as a random variable, follows Uniform distribution over $(0,1)$ and this result is basic to the inverse method of random variables generation. This distribution is also applied to determine power functions of tests of randomness. It is also applied in a power comparison of tests of non random clustering. There are also numerous applications in non parametric inference, such as Kolmogrov-Smirnov test for goodness of fit. It is well known that Uniform distribution can be used as a representation distribution of round-off errors, and it is also connected to probability integral transformations. Ristic and Popovic (2000a, b) introduced and studied the properties of a first order autoregressive $(\mathrm{AR}(1))$ time series model and discussed the parameter estimation of the uniform AR(1) process. Jose and Krishna (2011) introduced Marshall-Olkin extended uniform distribution as a generalization of uniform distribution and studied its properties.

Marshall and Olkin (1997) introduced a new family of distributions by adding a parameter to a given family of distributions. They started with a parent survival function $\bar{F}(x):=1-F(x)$ and considered a family of survival functions given by

$$
\bar{G}(x)=\frac{\alpha \bar{F}(x)}{F(x)+\alpha \bar{F}(x)}, \quad \alpha>0 .
$$

They constructed their family of distributions in the following way. Let $X_{1}, X_{2}, \ldots$. be a sequence of independent and identically distributed (i.i.d) random variables with survival function $\bar{F}(x)$.Let $\mathrm{N}$ be a geometric random variable with probability mass function (p.m.f) $\operatorname{Pr}(N=n)=\alpha(1-\alpha)^{n-1}$, for $n=1,2, \ldots$ and $0<\alpha<1$. Then the random variable $\mathrm{U}_{\mathrm{N}}=\min \left\{X_{1}, X_{2}, \ldots, X_{N}\right\}$ has the survival function given by (1). If $\alpha>1$ and $N$ is a geometric random variable with p.m.f 
$\operatorname{Pr}(N=n)=\frac{1}{\alpha}\left(1-\frac{1}{\alpha}\right)^{n-1}, n=1,2, \ldots$ then the random variable $\mathrm{V}_{\mathrm{N}}=\max \left\{X_{1}, X_{2}, . . X_{N}\right\}$ also has the survival function as in (1).

Many authors have studied various univariate distributions belonging to the Marshall-Olkin family of distributions; see Ristic et al. (2007), Jose et al. (2010) and Cordeioro and Lemente (2013). Jayakumar and Thomas (2008) proposed a generalization of the family of Marshall-Olkin distribution as

$$
\bar{G}(x ; \alpha, \gamma)=\left[\frac{\alpha \bar{F}(x)}{1-(1-\alpha) \bar{F}(x)}\right]^{\gamma}, \text { for } \alpha>0, \gamma>0 \text { and } x \in R
$$

Nadarajah et al. (2013) introduced a new family of life time models as follows:

Let $X_{1}, X_{2}$, .. be a sequence of independent and identically distributed random variables with survival function $\bar{F}(x)$. Let $N$ be a truncated negative binomial random variable with parameters $\alpha \in(0,1)$ and $\theta>0$.

That is,

$$
\operatorname{Pr}(N=n)=\frac{\alpha^{\theta}}{1-\alpha^{\theta}}\left(\begin{array}{c}
\theta+n-1 \\
\theta-1
\end{array}\right)(1-\alpha)^{n}, n=1,2, \ldots
$$

Consider $U_{N}=\min \left\{X_{1}, X_{2}, \ldots, X_{N}\right\}$. Then,

$$
\begin{aligned}
& \operatorname{Pr}\left(U_{N}>x\right)=\bar{G}_{U}(x) \\
= & \frac{\alpha^{\theta}}{1-\alpha^{\theta}} \sum_{n=1}^{\infty}\left(\begin{array}{c}
\theta+n-1 \\
\theta-1
\end{array}\right)((-\alpha) \bar{F}(x))^{n} .
\end{aligned}
$$

That is,

$$
\bar{G}_{U}(x)=\frac{\alpha^{\theta}}{1-\alpha^{\theta}}\left[(F(x)+\alpha \bar{F}(x))^{-\theta}-1\right]
$$

Similarly, if $\alpha>1$ and $N$ is a truncated negative binomial random variable with parameters $1 / \alpha$ and $\theta>0$, then $V_{N}=$ $\max \left\{X_{1}, X_{2}, \ldots, X_{N}\right\}$ also has the survival function (3).

This implies that we can consider a new family of distributions given by the survival function

$$
\begin{gathered}
\bar{G}_{U}(x ; \alpha, \theta)=\frac{\alpha^{\theta}}{1-\alpha^{\theta}}\left[(F(x)+\alpha \bar{F}(x))^{-\theta}-1\right], \\
\alpha>0, \theta>0 \text { and } x \in R .
\end{gathered}
$$

Note that $\bar{G}_{U}(x ; \alpha, \theta) \rightarrow \bar{F}(x)$ as $\alpha \rightarrow 1$. This family of distributions is a generalization of the Marshall-Olkin family, in the sense that the family is reduced to the Marshall-Olkin family of distributions, when $\theta=1$.

The aim of this paper is to introduce a new family of univariate distributions by using discrete Mittag-Leffler truncated distribution. In section 2 we introduce a new family of univariate distributions for a given parent distribution function F. This family contains the well-known MarshallOlkin family of distributions. We study some properties of this family, including random variate generation. In section3, we introduce a new family of univariate distribution which contains Uniform distribution and Marshall-Olkin extended Uniform distribution. We derive its shape properties, moments, median, mode, quantiles, distribution of order statistics, entropies and estimation procedure. In section 4, we discuss the estimation of parameters of DMLU by the method of maximum likelihood. An application in autoregressive time series modeling is presented in Section 5. Conclusions are presented in Section 6.

\section{Truncated Discrete Mittag-Leffler Family of Distributions}

Pillai and Jayakumar (1995) introduced the discrete Mittag-Leffler distribution and studied its properties. The mathematical origin of the discrete Mittag-Leffler distribution can be described as follows:

Consider a sequence of independent Bernoulli trails in which the $k^{\text {th }}$ trail has probability of success $\alpha / k$ with $0<\alpha<1$ and $k=1,2,3, \ldots$ Let $N$ be the trail number in which the first success occurs. Then the probability that $\{N=r\}$ is given by

$$
\begin{aligned}
\mathrm{p}_{\mathrm{r}} & =(1-\alpha)\left(1-\frac{\alpha}{2}\right)\left(1-\frac{\alpha}{3}\right) \ldots\left(1-\frac{\alpha}{\mathrm{r}-1}\right) \frac{\alpha}{\mathrm{r}} \\
& =\frac{(-1)^{r} \alpha(\alpha-1)(\alpha-2) \ldots(\alpha-r+1)}{r !} .
\end{aligned}
$$

Probability generating function (pgf) of $N$ is given by $G(z)=1-(1-z)^{\alpha}$. Let $X_{1}, X_{2}, \ldots, X_{n}$ be independent and identically distributed random variables as $N$ and let $X_{0}=0$. Let $M$ be geometric distributed random variable with parameter $p$, ie. $\operatorname{Pr}(M=k)=q^{k} p, k=0,1,2, \ldots ; 0<p<1, q=1-p$.

Then $X_{1}+X_{2}+\ldots+X_{M}$ has generating function

$$
P(z)=\frac{p}{1-q\left(1-(1-z)^{\alpha}\right)}=\frac{1}{1+c(1-z)^{\alpha}}
$$

with $\mathrm{p}=1 /(1+\mathrm{c})$. The distribution with $\operatorname{pgf}(5)$ is known as Discrete Mittag-Leffler distribution with parameters $\alpha$ and $c$. Define now a new random variable $Y$ such that.

$$
P(Y=x)=\frac{P(X=x)}{1-p_{0}}, x=1,2,3, \ldots
$$

Then

$$
\begin{aligned}
H(s) & =E\left(s^{Y}\right)=\sum_{y=1}^{\infty} \frac{s^{y} p(X=y)}{1-p_{0}} \\
& =\frac{1}{1-p_{0}}\left[\sum_{y=0}^{\infty} s^{y} p(X=y)-p(X=0)\right] \\
& =\frac{1}{1-p_{0}}\left[P(s)-\frac{1}{1+c}\right] \\
& =\frac{(1+c)}{c}\left[\frac{1}{1+c(1-s)^{\alpha}}\right]-\frac{1}{c} .
\end{aligned}
$$

Therefore,

$$
H(\bar{F}(x))=\frac{(1+c)}{c}\left[\frac{1}{1+c(1-\bar{F}(x))^{\alpha}}\right]-\frac{1}{c} .
$$

Hence we obtain a new family of distributions with 
parameters $\alpha$ and $c$ having survival function

$$
\bar{G}(x)=\frac{1-F^{\alpha}(x)}{1+c F^{\alpha}(x)} .
$$

The corresponding distribution function is given by

$$
G(x)=\frac{(1+c) F^{\alpha}(x)}{1+c F^{\alpha}(x)}
$$

Since discrete Mittag-Leffler distribution can be considered as a generalization of geometric distribution, the family of distributions given by (7) can be considered appropriate for life time modeling.

From (7), the p.d.f is given by

$$
g(x ; \alpha, c)=\frac{\alpha(1+c) F^{\alpha-1}(x) f(x)}{\left[1+c F^{\alpha}(x)\right]^{2}}, \alpha>0, c>0 x \in R
$$

where $f(x)$ is the p.d.f of $F(x)$, while the hazard rate function is given by

$$
h(x)=\frac{g(x)}{\bar{G}(x)}=\frac{\alpha(1+c) F^{\alpha-1}(x) f(x)}{\left[1-F^{\alpha}(x)\right]\left[1+c F^{\alpha}(x)\right.}
$$

The distribution belonging to (7) can easily be simulated. For a given parent distribution function $F$, random variable $X$ with distribution function (7) can be simulated as

$$
X=F^{-1}\left[\frac{Y}{1+c-c Y}\right]^{\frac{1}{\alpha}}
$$

where $Y$ is uniformly distributed random variable on $(0,1)$.

The newly constructed truncated Discrete Mittag-Leffler distribution can be considered as a generalization of Marshall-Olkin family of distributions since it reduces to Marshall-Olkin family when $\alpha=1$.

In (7), when $F(x)$ is exponential, $G(x)$ becomes the Marshall-Olkin generalized exponential distribution studied in Ristic and Kundu (2015). When $F(x)$ in (7) is Weibull, $G(x)$ reduces to Marshall-Olkin exponentiated Weibull distribution studied in Bidram et al. (2015). Hence (7) is a rich class in the sense that it leads to various generalizations of existing distributions that have the capability of modeling real data sets.

\section{A New Family of Uniform Distribution}

\subsection{Distribution Function}

Let $\mathrm{X} \sim \operatorname{Uniform}(0, \theta)$ distribution, where $\theta>0$. Then

$$
F(x)=\frac{x}{\theta}, \quad 0<x<\theta
$$

Using (7), we get the distribution function $G(x)$, for $F(x)$ in (11) as

$$
G(x)=\frac{(1+c) x^{\alpha}}{\theta^{\alpha}+c x^{\alpha}}
$$

We refer to this distribution as truncated Discrete MittagLeffler Uniform Distribution (DMLU) with parameters $\alpha, c$ and $\theta$; and write it as $\operatorname{DMLU}(\alpha, c, \theta)$.

The graph of $G(x)$ for different values of $\alpha$ and $c$ for $\theta=10$ is given in Figure1.

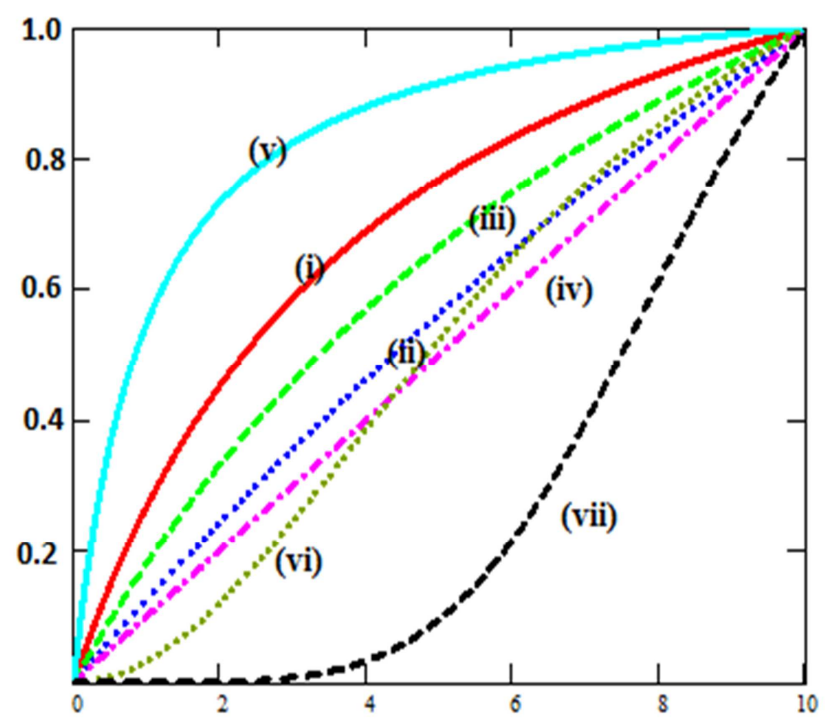

Figure 1. Distribution function of truncated Discrete Mittag-Leffler Uniform Distribution.(i) $\alpha=1, c=2.33$ (ii) $\alpha=1, c=3$ (iii) $\alpha=1, c=1$ (iv) $\alpha=1, c=0.01$ (v) $\alpha=1, c=10$ (vi) $\alpha=2, c=2.33$ (vii) $\alpha=5, c=2.33$.

\subsection{Probability Density Function}

The probability density function is given by

$$
g(x ; \alpha, c, \theta)=\frac{\alpha \theta^{\alpha}(1+c) x^{\alpha-1}}{\left[\theta^{\alpha}+c x^{\alpha}\right]^{2}}
$$

for $0<x<\theta, \alpha>0, c>0$ and $\theta>0$.

The graph of $g(x)$ for different values of $\alpha$ for $c=2.33$ and $\theta=10$ are given in Figure 2 .

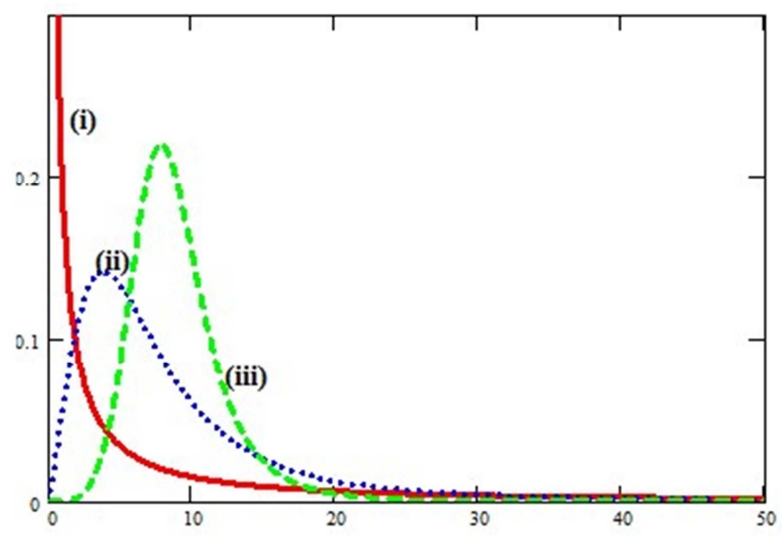

Figure 2. Probability density function of $\operatorname{DMLU}(\alpha, 2.33,10)$ (i) $\alpha=0.5$ (ii) $\alpha$ $=1.5$ (iii) $\alpha=2.0$.

Some special cases of the $\operatorname{DMLU}(\alpha, c, \theta)$ are:

Case I: When $\alpha=1, c=q / p$

$$
g\left(x ; 1, \frac{q}{p}, \theta\right)=\frac{p \theta}{\frac{[p \theta+q x]^{2}}{0<x<\theta, 0<p<1, \quad q=1-p}}
$$

This is Marshall-Olkin Extended Uniform (MOEU) distribution studied in Jose and Krishna (2011).

Case II: When $\alpha=1$ and $\mathrm{c} \rightarrow 0$ 


$$
g(x ; 1,0, \theta)=\frac{1}{\theta}, \quad 0<x<\theta .
$$

which is Uniform distribution in $(0, \theta)$.

In order to derive the shape properties of the p.d.f (13), we consider the function

$$
(\log g)^{\prime}=\frac{(\alpha-1)\left(\theta^{\alpha}+c x^{\alpha}\right)-2 c \alpha x^{\alpha}}{x\left(\theta^{\alpha}+c x^{\alpha}\right)}
$$

The following shapes are possible:

1 Let $\alpha \in(0,1)$. Then $g(x)$ is a decreasing function with $g(0)=\infty$ and $g(\theta)=\frac{\alpha}{\theta(1+c)}$.

2 Let $\alpha>1$. Then $g(x)$ is a unimodal function with mode at $x_{0}$. Furthermore $g(0)=0$ and $g(\theta)=\frac{\alpha}{\theta(1+c)}$.

\subsection{Hazard Rate Function}

The hazard rate function is given by

$$
h(x)=\frac{\theta^{\alpha} \alpha(1+c) x^{\alpha-1}}{\left(\theta^{\alpha}-x^{\alpha}\right)\left(\theta^{\alpha}+c x^{\alpha}\right)}
$$

The shapes of hazard rate function varies with respect to $\alpha$ :

1. If $0<\alpha<1$, then $h(x)$ initially decreasing and then moving constantly and then increasing steeply.

2. If $\alpha>1, h(x)$ is moving constantly at the initial values and then increasing steeply. The graph of hazard function for different values for $\alpha$ and $c$ when $\theta=10$ are given in Figure 3.

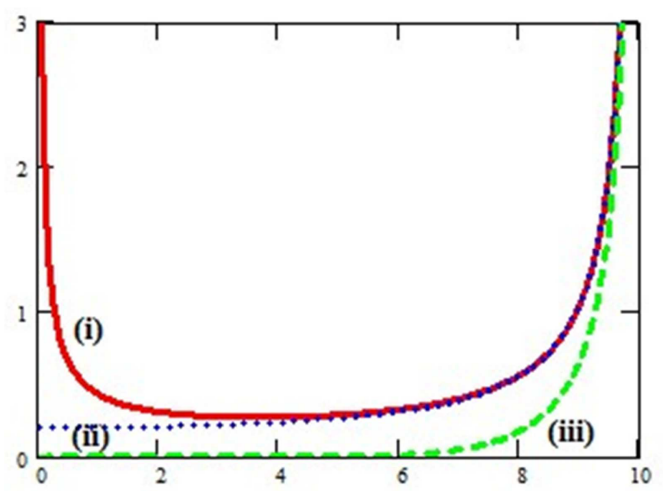

Figure 3. Hazard rate function of $D M L U(\alpha, c, 10)$ (i) $\alpha=0.1, c=1$ (ii) $\alpha=1, c$ $=1$ (iii) $\alpha=10, c=2$.

\subsection{Moments}

Suppose that $X$ has the $\operatorname{DMLU}(\alpha, c, \theta)$. The $\mathrm{r}^{\text {th }}$ moment can be written as

$$
E\left(X^{r}\right)=\alpha \theta^{\alpha}(1+c) \int_{0}^{\theta} \frac{x^{r+\alpha-1}}{\left(\theta^{\alpha}+c x^{\alpha}\right)^{2}} d x
$$

Let $\mathrm{x}^{\alpha}=\mathrm{u}$, above equation reduces to

$$
E\left(X^{r}\right)=\theta^{\alpha}(1+c) \int_{0}^{\theta^{\alpha}} \frac{u^{\frac{r}{\alpha}}}{\left(\theta^{\alpha}+c u\right)^{2}} d u .
$$

From Prudnikov et al. (1986), equation (2.2.5.2) is

$$
\begin{gathered}
\int_{a}^{b} \frac{(x-a)^{\alpha-1}}{(c x+d)^{\alpha+n+1}} d x \\
=\frac{(b-a)^{\alpha}}{(a c+d)(b c+d)^{\alpha}} \sum_{k=0}^{n}\left(\begin{array}{l}
n \\
k
\end{array}\right) \frac{B(\alpha+k, n-k+1)}{(b c+d)^{k}(a c+d)^{n-k}},
\end{gathered}
$$

where a, b, c and d are real numbers with $(\mathrm{ac}+\mathrm{d})(\mathrm{bc}+\mathrm{d})>0$; Real part of $\alpha>0$ and $\mathrm{B}(\mathrm{a}, \mathrm{b})=\frac{\Gamma(a) \Gamma(b)}{\Gamma(a+b)}$. Hence if $r / \alpha$ is a positive integer, we have,

$$
\begin{gathered}
\int_{0}^{\theta^{\alpha}} \frac{u^{\frac{r}{\alpha}}}{\left(\theta^{\alpha}+c u\right)^{2}} d u \\
=\frac{1}{\theta^{r+\alpha}(1+c)^{1+\frac{r}{\alpha}}} \sum_{k=0}^{r / \alpha}\left(\begin{array}{c}
r / \alpha \\
k
\end{array}\right) \frac{B\left(1+k+\frac{r}{\alpha^{\prime}} 1-k+\frac{r}{\alpha}\right)}{(1+c)^{k}}
\end{gathered}
$$

Therefore

$$
E\left(X^{r}\right)=\frac{1}{\theta^{r}(1+c)^{\frac{r}{\alpha}}} \sum_{k=0}^{r / \alpha}\left(\begin{array}{c}
r / \alpha \\
k
\end{array}\right) \frac{B\left(1+k+\frac{r}{\alpha^{1}} 1-k+\frac{r}{\alpha}\right)}{(1+c)^{k}}
$$

In particular,

$$
\begin{gathered}
E(X)=\frac{1}{\theta(1+c)^{\frac{1}{\alpha}}} \sum_{k=0}^{1 / \alpha}\left(\begin{array}{c}
1 / \alpha \\
k
\end{array}\right) \frac{\Gamma\left(1+k+\frac{1}{\alpha}\right) \Gamma\left(1-k+\frac{1}{\alpha}\right)}{\Gamma 2\left(1+\frac{1}{\alpha}\right)(1+c)^{k}} \\
E\left(X^{2}\right)=\frac{1}{\theta(1+c)^{\frac{2}{\alpha}}} \sum_{k=0}^{2 / \alpha}\left(\begin{array}{c}
2 / \alpha \\
k
\end{array}\right) \frac{\Gamma\left(1+k+\frac{2}{\alpha}\right) \Gamma\left(1-k+\frac{2}{\alpha}\right)}{\Gamma 2\left(1+\frac{2}{\alpha}\right)(1+c)^{k}}
\end{gathered}
$$

$\operatorname{Var}(X)=E\left(X^{2}\right)-E(X)^{2}$.

The q-th quantile of a random variable $X$ following $\operatorname{DMLU}(\alpha, c, \theta)$ is given through the quantile function as

$$
x_{q}=G^{-1}(q)=\left[\frac{q \theta^{\alpha}}{c+1-q}\right]^{\frac{1}{\alpha}}, 0 \leq q \leq 1,
$$

where $G^{-1}($.$) denote the inverse distribution function of G($.$) .$ In particular, the median of $X$ is given by

$$
\text { Median }=\frac{\theta}{(2 c+1)^{\frac{1}{\alpha}}} .
$$

Finally, the mode of $X$ is given by

$$
\text { Mode }=\theta\left[\frac{\alpha-1}{c(\alpha+1)}\right]^{\frac{1}{\alpha}}
$$

The mean and variance of DMLU for different values of $\alpha$ and $c$ when $\theta=10$ are calculated (via MATHCAD) and given in Table 1. 
Table 1. Mean and Variance of DMLU for different values of $\alpha$ and $c$ when $\theta=10$.

\begin{tabular}{llllllllllll}
\hline $\mathbf{c} \downarrow$ & $\boldsymbol{\alpha \rightarrow}$ & $\mathbf{0 . 1}$ & $\mathbf{0 . 2}$ & $\mathbf{0 . 4}$ & $\mathbf{0 . 5}$ & $\mathbf{1 . 0}$ & $\mathbf{2 . 0}$ & $\mathbf{5 . 0}$ & $\mathbf{1 0 . 0}$ & $\mathbf{1 5 . 0}$ & $\mathbf{2 0 . 0}$ \\
\hline \multirow{2}{*}{0.0} & Mean & 0.91 & 1.67 & 2.86 & 3.33 & 5.0 & 6.67 & 8.33 & 9.09 & 9.38 & 9.52 \\
& Variance & 3.93 & 6.31 & 8.51 & 8.89 & 8.33 & 5.55 & 1.81 & 0.69 & 0.34 & 0.20 \\
0.25 & Mean & 0.75 & 1.42 & 2.51 & 2.97 & 4.63 & 6.37 & 8.16 & 8.99 & 9.31 & 9.47 \\
& Variance & 3.31 & 5.52 & 7.84 & 8.35 & 8.28 & 5.77 & 2.14 & 0.76 & 0.38 & 0.23 \\
\multirow{2}{*}{1.0} & Mean & 0.50 & 0.98 & 1.87 & 2.27 & 3.86 & 5.08 & 7.67 & 8.76 & 9.14 & 9.35 \\
& Variance & 2.25 & 4.13 & 6.31 & 6.98 & 7.82 & 12.84 & 2.45 & 0.89 & 0.45 & 0.27 \\
\multirow{2}{*}{1.5} & Mean & 0.41 & 0.82 & 1.61 & 1.98 & 3.51 & 5.39 & 7.57 & 8.64 & 9.06 & 9.28 \\
& Variance & 1.85 & 3.39 & 5.57 & 6.28 & 7.46 & 6.08 & 2.57 & 0.95 & 0.49 & 0.29 \\
4.0 & Mean & 0.21 & 0.45 & 0.96 & 1.24 & 2.53 & 4.42 & 6.92 & 8.25 & 8.78 & 9.06 \\
& Variance & 0.99 & 1.92 & 3.54 & 4.19 & 5.96 & 5.76 & 2.84 & 1.12 & 0.59 & 0.36 \\
9.0 & Mean & 0.11 & 0.24 & 0.55 & 0.73 & 1.73 & 3.52 & 6.25 & 7.83 & 8.47 & 8.82 \\
& Variance & 0.51 & 1.03 & 2.06 & 2.53 & 4.26 & 4.96 & 2.90 & 1.24 & 0.66 & 0.42 \\
\hline
\end{tabular}

\subsection{Order Statistics}

Assume $X_{1}, X_{2}, \ldots, X_{n}$ are independent random variables having the $\operatorname{DMLU}(\alpha, c, \theta)$ distribution. Let $X_{i: n}$ denote the $\mathrm{i}^{\text {th }}$ order statistic. The p.d.f of $X_{i: n}$ is

$$
\begin{aligned}
g_{i: n}(x ; \alpha, c, \theta) & =\frac{n !}{(i-1) !(n-1) !} g(x ; \alpha, c, \theta) G^{i-1}(x ; \alpha, c, \theta) \bar{G}^{n-i}(x ; \alpha, c, \theta) \\
& =\frac{(n !) \alpha \theta^{\alpha}(1+c)^{i} x^{\alpha i-1}\left(\theta^{\alpha}-x^{\alpha}\right)^{n-i}}{(i-1) !(n-i) !\left(\theta^{\alpha}+c x^{\alpha}\right)^{n+i}}
\end{aligned}
$$

\subsection{Renyi and Shannon Entropies}

Entropy is, in principle, a measure of variation or uncertainty. The Renyi entropy of a random variable with p.d.f $g($.$) is$ defined as

$$
I_{R}(\gamma)=\frac{1}{1-\gamma} \int_{0}^{\infty} g^{\gamma}(x) d x . \gamma>0, \gamma \neq 1 .
$$

The Shannon entropy of a random variable $X$ is defined by $\mathrm{E}[-\log \mathrm{g}(X)]$. It is the particular (limiting) case of the Renyi entropy for $\gamma \rightarrow 1$. Let us first derive the Renyi entropy. We have

$$
\int_{0}^{\infty} g^{\gamma}(x) d x=\left[\alpha \theta^{\alpha}(1+c)\right]^{\gamma} \int_{0}^{\infty}\left[\frac{x^{\alpha-1}}{\left(\theta^{\alpha}+c x^{\alpha}\right)^{2}}\right]^{\gamma} d x=\left[\alpha \theta^{\alpha}(1+c)\right]^{\gamma} \int_{0}^{\infty}\left[\frac{x^{\gamma(\alpha-1)}}{\left(\theta^{\alpha}+c x^{\alpha}\right)^{2 \gamma}}\right] d x
$$

Let $\mathrm{u}=\mathrm{x}^{\alpha}$. Then

$$
\int_{0}^{\infty}\left[\frac{x^{\gamma(\alpha-1)}}{\left(\theta^{\alpha}+c x^{\alpha}\right)^{2 \gamma}}\right] d x=\frac{1}{\alpha} \int_{0}^{\infty} \frac{u^{\frac{1}{\alpha}[\gamma(\alpha-1)-\alpha+1]}}{\left(\theta^{\alpha}+c u\right)^{2 \gamma}} d u .
$$

Using eq.(2.2.5.2) from Prudnikov et al. (1986) and if $\gamma-\frac{1}{\alpha}(1-\gamma-\alpha)$ is a positive integer, the above integral becomes

$$
\frac{1}{\theta^{\alpha}(1+c)^{\frac{1}{\alpha}[\gamma(\alpha-1)-2 \alpha+1]}} \sum_{k=0}^{\gamma-\frac{1}{\alpha}(1-\gamma-\alpha)} \frac{B\left(\frac{1}{\alpha}[\gamma(\alpha-1)-\alpha+1]+k-1, \gamma-\frac{1}{\alpha}(1-\gamma-\alpha)-k-1\right)}{(1+c)^{k} \theta^{\gamma \alpha+\gamma+\alpha-1}}
$$

Therefore the Renyi entropy is

$$
\begin{gathered}
I_{R}(\gamma)=\frac{1}{1-\gamma} \log \left[\alpha \theta^{\alpha}+(1+c)\right]^{\gamma} \frac{1}{\theta^{\alpha}(1+c)^{\frac{1}{\alpha}[\gamma(\alpha-1)-2 \alpha+1]}} \\
\sum_{k=0}^{\gamma-\frac{1}{\alpha}(1-\gamma-\alpha)} \frac{B\left(\frac{1}{\alpha}[\gamma(\alpha-1)-\alpha+1]+k-1, \gamma-\frac{1}{\alpha}(1-\gamma-\alpha)-k-1\right)}{(1+c)^{k} \theta^{\gamma \alpha+\gamma+\alpha-1}} .
\end{gathered}
$$

The Shannon entropy is

$$
E[-\log g(X)]=-\log \left[\alpha \theta^{\alpha}(1+c)\right]-(\alpha-1) E[\log (X)]+2 E\left[\log \left(\theta^{\alpha}+c x^{\alpha}\right)\right] .
$$




\section{Estimation}

Since the moments of a DMLU random variable cannot be obtained in closed from, we consider estimation of the unknown parameters by the method of maximum likelihood. For a given sample $\mathbf{X}:=\left(x_{1}, x_{2}, \ldots, x_{n}\right)$, the log-likelihood function is given by

$$
\begin{aligned}
\log L(x ; \alpha, c, \theta) & =n \log \left[\alpha \theta^{\alpha}(1+c)\right]+(\alpha-1) \sum_{i} \log \left(x_{i}\right) \\
& \left.-2 \sum_{i} \log \left(\theta^{\alpha}+c x_{i}^{\alpha}\right)\right] \\
=n \log \alpha+n \alpha & \log \theta+n \log (1+c)+(\alpha-1) \sum_{i} \log \left(x_{i}\right) \\
& \left.-2 \sum_{i} \log \left(\theta^{\alpha}+c x_{i}^{\alpha}\right)\right] .
\end{aligned}
$$

The partial derivatives of the log-likelihood function with respect to the parameters are

$$
\begin{gathered}
\frac{\partial \log L}{\partial \alpha}=\frac{n}{\alpha}+n \log \theta+\sum_{i} \log \left(x_{i}\right)-2 \sum_{i} \frac{\log \left(\theta+c x_{i}\right)}{\left(\theta^{\alpha}+c x_{i}^{\alpha}\right)} \\
\frac{\partial \log L}{\partial c}=\frac{n}{(1+c)}-2 \sum_{i} \frac{x_{i}^{\alpha}}{\left(\theta^{\alpha}+c x_{i}^{\alpha}\right)} \\
\frac{\partial \log L}{\partial \theta}=\frac{n \alpha}{\theta}-2 \sum_{i} \frac{\alpha \theta^{\alpha-1}}{\left(\theta^{\alpha}+c x_{i}^{\alpha}\right)} .
\end{gathered}
$$

The maximum likelihood estimates can be obtained numerically solving the equation. $\frac{\partial \log L}{\partial \alpha}=0, \frac{\partial \log L}{\partial c}=0$, $\frac{\partial \log L}{\partial \theta}=0$. We can use, for example, the function $\mathrm{nlm}$ from the programming language $\mathrm{R}$.

The second derivatives of the log-likelihood function of DMLU with respect to $\alpha, c$ and $\theta$ are given by

$$
\begin{aligned}
& \frac{\partial^{2} \log L}{\partial \alpha^{2}}=-\frac{n}{\alpha^{2}}+2 \sum_{i}\left(\theta^{\alpha}+c x_{i}^{\alpha}\right) \log \alpha \log \left(\theta+c x_{i}\right), \\
& \frac{\partial^{2} \log L}{\partial c^{2}}=-\frac{n}{(1+c)^{2}}+2 \sum_{i} \frac{x_{i}^{2 \alpha}}{\left(\theta^{\alpha}+c x_{i}^{\alpha}\right)^{2}}, \\
& \frac{\partial^{2} \log L}{\partial \theta^{2}}=-\frac{n \alpha}{\theta^{2}}-2 \alpha \sum_{i} \frac{\left[(\alpha-1) c x_{i}^{\alpha} \theta^{\alpha-2}-\theta^{2(\alpha-1)}\right.}{\left(\theta^{\alpha}+c x_{i}^{\alpha}\right)^{2}}, \\
& \frac{\partial^{2} \log L}{\partial \alpha \partial \theta}=\frac{n}{\theta}-2 \sum_{i} \frac{\left[\left(\theta^{\alpha}+c x_{i}^{\alpha}\right)-2 \theta\left(\theta+c x_{i}\right) \log \left(\theta+c x_{i}\right)\right]}{\left(\theta+c x_{i}\right)\left(\theta^{\alpha}+c x_{i}^{\alpha}\right)^{2}}, \\
& \frac{\partial^{2} \log L}{\partial \alpha \partial c}=-2 \sum_{i} \frac{\left[x_{i}\left(\theta^{\alpha}+c x_{i}^{\alpha}\right)-x_{i}^{\alpha}\left(\theta+c x_{i}\right) \log \left(\theta+c x_{i}\right)\right]}{\left(\theta+c x_{i}\right)\left(\theta^{\alpha}+c x_{i}^{\alpha}\right)^{2}}, \\
& \frac{\partial^{2} \log L}{\partial \theta \partial c}=2 \sum_{i} \frac{\alpha \theta^{\alpha-1} x_{i}^{\alpha}}{\left(\theta^{\alpha}+c x_{i}^{\alpha}\right)^{2}} .
\end{aligned}
$$

If we denote the MLE of $\boldsymbol{\beta}:=(\alpha, c, \theta)$ by, $\widehat{\boldsymbol{\beta}}=(\hat{\alpha}, \hat{c}, \widehat{\theta})$, then the observed information matrix is given by

$$
I(\boldsymbol{\beta})=-E\left[\begin{array}{ccc}
\frac{\partial^{2} \log L}{\partial \alpha^{2}} & \frac{\partial^{2} \log L}{\partial \alpha \partial c} & \frac{\partial^{2} \log L}{\partial \alpha \partial \theta} \\
\frac{\partial^{2} \log L}{\partial \alpha \partial c} & \frac{\partial^{2} \log L}{\partial c^{2}} & \frac{\partial^{2} \log L}{\partial \theta \partial c} \\
\frac{\partial^{2} \log L}{\partial \alpha \partial \theta} & \frac{\partial^{2} \log L}{\partial \theta \partial c} & \frac{\partial^{2} \log L}{\partial \theta^{2}}
\end{array}\right]
$$

and hence the variance covariance matrix of the estimate vector $\widehat{\boldsymbol{\beta}}$ would be $\mathbf{I}^{-\mathbf{1}}(\widehat{\boldsymbol{\beta}})$.

The approximate $(1-\delta) \%$ confidence intervals for the parameters $\alpha, \mathrm{c}, \theta$ are $\hat{\alpha} \pm Z_{\frac{\delta}{2}} \sqrt{V(\hat{\alpha})}, \hat{c} \pm Z_{\frac{\delta}{2}} \sqrt{V(\hat{c})}, \hat{\theta} \pm$ $Z_{\frac{\delta}{2}} \sqrt{V(\hat{\theta})}$ respectively, where $\mathrm{V}(\hat{\alpha}), \mathrm{V}(\hat{c})$ and. $\mathrm{V}(\hat{\theta})$ are the variances of $\hat{\alpha}, \hat{c}$ and $\hat{\theta}$, which are given by the diagonal elements of $\mathrm{I}^{-1}(\beta)$, and $Z_{\frac{\delta}{2}}$ is the upper $\left(\frac{\delta}{2}\right)$ percentile of standard normal distribution.

\section{Autoregressive Time Series Modeling}

Time series models with uniform marginal distribution are studied by various researchers (see Ristic and Popovic (2000a,b) and Jose and Krishna (2011)). Here we develop an AR(1) model with DMLU as marginal distribution, which is considered as a generalization of the existing time series models with uniform marginal.

Consider the $\mathrm{AR}(1)$ process

$$
X_{n}=\left\{\begin{array}{l}
\epsilon_{n} \text { with probability } \alpha \\
\min \left(X_{n-1}, \epsilon_{n}\right) \text { with probability } 1-\alpha,
\end{array}\right.
$$

where $0 \leq \alpha \leq 1, n \geq 1$ and $\left\{\epsilon_{n}\right\}$ is a sequence of i.i.d random variables with power function distribution in $(0, \theta)$.

Theorem 5.1: Consider the AR(1) structure given in (21) with $X_{0}$ distributed as a $\operatorname{DMLU}(\alpha, c, \theta)$ distribution. Let $c=\frac{1-\alpha}{\alpha}$. Then $\left\{\mathrm{X}_{\mathrm{n}}, \mathrm{n} \geq 1\right\}$ is a stationary Markovian autoregressive model with $\operatorname{DMLU}(\alpha, \mathrm{c}, \theta)$ marginals iff $\left\{\epsilon_{n}\right\}$ is distributed as power distribution in $(0, \theta)$ with survival function $1-\left(\frac{x}{\theta}\right)^{\alpha}$.

Proof: From the above expression (21), it follows that

$$
\bar{F}_{X_{n}}(x)=\alpha \bar{F}_{\epsilon_{n}}(x)+(1-\alpha) \bar{F}_{X_{n-1}}(x) \bar{F}_{\epsilon_{n}}(x)
$$

Using the fact that $X_{0}$ has $\operatorname{DMLU}(\alpha, \theta)$ distribution and $\epsilon_{1}$ has a power distribution in $(0, \theta)$ with distribution function $\left(\frac{x}{\theta}\right)^{\alpha}$,we obtain, that for $n=1$,

$$
\begin{gathered}
\bar{F}_{X_{1}}(x)=\left[\alpha+(1-\alpha) \bar{F}_{X_{0}}(x)\right] \bar{F}_{\epsilon_{1}}(x) \\
=\left[\alpha+(1-\alpha) \frac{\theta^{\alpha}-x^{\alpha}}{\theta^{\alpha}+\left(\frac{1-\alpha}{\alpha}\right) x^{\alpha}}\right]\left[1-\left(\frac{x}{\theta}\right)^{\alpha}\right] \\
=\frac{\theta^{\alpha}-x^{\alpha}}{\theta^{\alpha}+\left(\frac{1-\alpha}{\alpha}\right) x^{\alpha}}=\frac{\theta^{\alpha}-x^{\alpha}}{\theta^{\alpha}+c x^{\alpha}} .
\end{gathered}
$$


which means that $X_{l}$ has $\operatorname{DMLU}(\alpha, \mathrm{c}, \theta)$ distribution where $c=\frac{1-\alpha}{\alpha}$. Assume that $X_{n-1}$ is distributed as $\operatorname{DMLU}(\alpha, \mathrm{c}, \theta)$. Then by induction method we can establish that $\left\{X_{n}\right\}$ is distributed as $\operatorname{DMLU}(\alpha, \mathrm{c}, \theta)$.

Hence the process $\left\{X_{n}\right\}$ is stationary.

Conversely, if $\left\{X_{n}, n \geq 1\right\}$ is stationary with $\operatorname{DMLU}(\alpha, \theta)$ marginal's, it can be easily shown that $\left\{\epsilon_{n}\right\}$ has power distribution, $F(x)=\left(\frac{x}{\theta}\right)^{\alpha}, 0<x<\theta$. Hence the theorem.

Even if $X_{0}$ is arbitrary, it is easy to establish that $\left\{X_{n}\right\}$ is asymptotically stationary.

\section{Conclusion}

Marshall and Olkin (1997) introduced a way of expanding a given family of distribution by adding a parameter. In this paper, we introduced the Discrete Mittag-Leffler truncated distribution as a generalization of Marshall-Olkin family of distributions and studied its properties. This class is a rich class in the sense that some of the recently investigated distributions are members of this family; see Ristic and Kundu (2015), and Bidram et al. (2015). As a particular case, a three parameter generalization of Uniform distribution was given special attention. The shape properties of the distribution were studied. The expression for moments, distributions of the order statistics and entropies were also derived. Moreover, we discussed the maximum likelihood method of estimation of the distribution's parameters. An application on the autoregressive time series modeling was also presented.

\section{References}

[1] H. Bidram, M. H. Alamastsaz and V. Nekoukhou, (2015). On an extension of the exponentiated Weibull distribution. Communications in Statistics - Simulation and Computation, 44, 1389-1404.
[2] G. M. Cordeiro and A. J. Lemonte, (2013). On the MarshallOlkin extended Weibull distribution. Statistical Papers, 54, 333-353.

[3] K. Jayakumar and M. Thomas, (2008). On a generalization to Marshall-Olkin scheme and its application to Burr type XII distribution. Statistical Papers, 49, 421-439.

[4] K. K. Jose, S. R. Naik and M. M. Ristic, (2010). MarshallOlkin q Weibull distribution and maximin processes. Statistical Papers, 51, 837-851.

[5] K. K. Jose and E. Krishna, (2011). Marshall-Olkin extended uniform distribution. ProbStat Forum, 04, 78-88.

[6] A. W. Marshall and I. Olkin, (1997). A new method for adding a parameter to a family of distributions with application to the exponential and Weibull families. Biometrika, 84, 641-652.

[7] S. Nadarajah, K. Jayakumar and M. M. Ristic, (2013). A new family of life- time models. Journal of Statistical Computation and Simulation, 83, 1389-1404.

[8] R. N. Pillai and K. Jayakumar, (1995). Discrete Mittag-Leffler distributions. Statistics and Probability Letters, 23, 271-274.

[9] A. P. Prudnikov, Y. A. Brychkov and O.I Marichev, (1986). Integrals and series Vol. I, Gordeon and Breach Sciences, Amsterdam, Netherlands.

[10] M. M. Ristic and B. C. Popovic, (2000 a). A new Uniform AR(1) time series Model (NUAR(1)). Publications De Linstitut Mathematique Nouvelle series, 68, 145-152.

[11] M. M. Ristic and B. C. Popovic, $(2000$ b). Parameter estimation for Uniform autoregressive processes. Novi Sad Journal of Mathematics, 30, 89-95.

[12] M. M. Ristic, K. K. Jose and A. Joseph, (2007). A MarshallOlkin gamma distribution and minification process. STARS International Journal (Science), 1, 107-117.

[13] M. M. Ristic and D. Kundu, (2015). Marshall-Olkin generalized exponential distribution. Metron, 73, 317-333. 